\title{
IMPACT OF HEAT STRESS ON GROWTH PERFORMANCE AND CARCASS TRAITS IN SOME BROILER CHICKENS
}

Omran O.M., Galal A., Mahrous M.Y. and Badri F.B.

Poultry Production Dept., Fac. of Agric., Ain Shams Univ., P.O. Box 68, Hadayek Shoubra 11241, Cairo, Egypt

${ }^{\star}$ Corresponding author: omeromran1987@gmail.com

Received 16 May, 2020

Accepted 19 August, 2020

\begin{abstract}
Environmental heat stress is one of the most challenging conditions in the world which have adverse impact on the industry of poultry. Broiler chicken strains are delicate to heat stress primarily due to not having sweat glands. The current study was aimed to investigate the effects of heat stress exposed on growth performance and Bio-physiological characteristics for (Cobb, Hubbard and Arbor Acres broiler hybrids) under the summer season when environmental conditions of Egypt were revealed. A total of three hundred one day old (one hundred birds from each hybrid) at one day of age were brooding under the same conditions of water, diet consumed, breeding system, vaccines and medications used during the period birds life even slaughtering age. The three strains were randomly divided into twelve groups (three strains "Cobb, Hubbard and Arbor Acres" X two treatments "control group and heat exposed group" $X$ two replicates $X$ twenty five chicks). The degrees of environmental temperature and relative humidity during housing are (Environmental temperature $=32^{\circ} \pm 2$ Celsius degree, Relative humidity $=50 \pm 5$ percentage) for control group and (Environmental temperature $=40^{\circ} \pm 2$ Celsius degree, Relative humidity $=20 \pm 5$ percentage) for the heat stressed group. The body weight, body weight gain, edible parts of carcass (carcass, thigh, drum, breast muscles and giblets weight) and inedible parts of carcass (blood, feathers, head and legs weight) were recorded to heat stressed group and control group. Lymphatic organs such as spleen, thymus and bursa weight were measured also. The last results concluded that the Cobb strain showed the best growth performance and carcass characteristics under heat
\end{abstract}

stress condition, while the Arbor Acres strain considered the best strain which didn't effected a lot in their rectal temperature such another strains with heat exposure. The Arbor Acres strain for each group (control and treated) have the highest viability. The control group and Hubbard strain showed an increase in bursa weight compared to heat exposed group and another strains. It was concluded that the Cobb strain has the best performance under heat stress comparing to the other strains broiler chicken.

Keywords: Broiler, Growth, Carcass and Heat exposure

\section{INTRODUCTION}

The poultry industry was major source protein to human so the poultry production was increased during last two decades. But the high ambient temperatures were negative effect of production traits. Pourreza and Edriss (1992) raised broiler at 20 or $30^{\circ} \mathrm{C}$ (control and high temperatures respectively) until 45 days of age, compared with normal temperatures high temperature decreased slaughter and carcass weights and increased dressing percentage. Broiler chickens are sensitive to heat stress (Yousaf et al 2019, Yousaf et al 2018). Poultry birds don't have sweat glands for heat releasing factor, if panting failed to decrease the high internal body temperature, birds become inactive, exhausted and mortality happened because of the circulatory, respiratory and electrolytes imbalance (Swayne, 2017). It has been exploring the impact of high environmental temperature on the performance of different poultry species, including broilers (Dozier et al 2007) and has found that high environmental temperatures have pernicious impacts on 
productive performance. Increase body temperature of poultry (Altan et al 2000), water consumption (Arce-Menocal et al 2009) and decrease of feed consumption due to higher ambient temperature (Dozier et al 2007). Humidity and temperature play a key role which is one of the foremost imperative environmental factors during poultry housing (Lourens et al 2005). Broilers expose to higher ambient temperature, increase body temperature (Reddy, 2000) consequently released the corticosterone hormone into the circulation of blood to help the metabolism (Arce-Menocal et al 2009). This hormone might cause humoral immunity and cell mediate failure cause of the changing's in plasma concentrations of Adrenocorticotropic Hormone and corticosteroids affect the lymphoid organs, decrease the mass of spleen, thymus and bursa (Havenstein et al 2003). The current study was pointed to investigate the impacts of adaptation of heat stress on growth performance and characteristics carcass.

\section{MATERIALS AND METHODS}

\section{Statement of the Experiment}

This experiment was carried out at Poultry Breeding Farm, Poultry Production Department, Faculty of Agriculture, Ain Shams University. A total of three hundred one day old chicks from three broiler strains (100 Cobb, 100 Hubbard and $100 \mathrm{Ar}-$ bor Acres). All groups were isolated from each other by plastic sheet barriers.

Broiler chicks were randomly divided into the following groups: Normal control group (C) exposed to environmental temperature $\left(T=32 \pm 2^{\circ} \mathrm{C}\right)$ at the day of hatch and decreased $1^{\circ} \mathrm{C}$ every three days even $16^{\text {th }}$ day of age when temperature stabled at $24^{\circ} \mathrm{C}$ even age of Slaughtering, while the relative humidity recorded arrange from (40-60\%) during the age of birds. Another group which exposed to heat stress had the same temperature program, but exposed to $40^{\circ} \mathrm{C}$ from 1 day up to 7 day of age for 3 hours daily and exposed to $40^{\circ} \mathrm{C}$ for 3 hours before slaughtering directly at 35 day of age.

\section{Housing and Management}

Before receiving the experimental chicks, the broiler house is used for rearing the chicks with density about 7 birds $/ \mathrm{m}^{2}$. All orifices of the rearing house (semi-open-housing system) had been closed to maintain temperature and a thermometer was installed to monitor temperature in the center of the room.

Feed and water were supplied ad libitum. Birds were fed on a commercial starter diet based on corn-soybean meal; containing $23 \%$, crude protein and $3000 \mathrm{kcal} / \mathrm{kg} \mathrm{ME}$ from one day to 17 days of age, and replaced thereafter by a diet containing $21 \%$, crude protein and $3050 \mathrm{kcal} / \mathrm{kg}$ grower diet which then have been substituted by a diet containing $19 \%$, crude protein and $3100 \mathrm{kcal} / \mathrm{kg}$.

\section{Body weight and body weight gain}

Body weight was recorded individually and weight gain calculated weekly to the nearest $(0.1 \mathrm{~g})$ throughout the experimental period at day 1 and from 1 week until 5 weeks of age.

\section{Slaughter test and carcass traits}

A total of 60 birds ( 20 chickens from each strain, 10 birds from each treatment) at the end of the growth experiment will be taken at 35 day of age randomly from each treatment. After complete bleeding was over, birds were scalded at $60 \mathrm{C}^{\circ}$ water for 30 seconds; feathers were removed by defeathering machine. After the removal of head, viscera, shanks, edible parts(gizzard, heart, and liver) the rest of the body was weighted to determine the dressed weight which includes the front parts with wings, hind parts and the neck. The dressed birds were portioned into right and left sides. The right side of each carcass was halved into a front quarter (breast and wings) and hindquarter (thigh and drumstick). The edible and non-edibles parts were also calculated. Also, lymphatic organs such as spleen, thymus and bursa were removed and weighed. After that the right side of each carcass was split into its cuts, breast, drumstick and thigh were weighted and recorded.

\section{Bio-physiological characteristics}

\section{Rectal Temperature (RT)}

Rectal Temperature was measured during the thermal challenge period in ten random birds for each treatment during the age from 1 up to 7 day of age through exposing to heat stress ( 5 birds for each replicate). Rectal temperature $\left( \pm 0.1^{\circ} \mathrm{C}\right)$ was measured by inserting a probe of electronic thermometer $3 \mathrm{~cm}$ into the colon. 


\section{Mortality Rate}

The number of dead individuals during the breeding period was individually recorded to evaluate the viability of three broiler strains under control and heat exposure conditions

\section{Statistical Analysis}

A general linear model of $S A S \AA$ was used for two-way analysis of variance of statistical analysis system (SAS 9.1.3., 2003). Factorial design (2X3) was used to examine the effects of different temperature on productive performance, physiological parameters and three strains to evaluate the acclimation of broiler chickens in response to thermal conditioning. When significant differences among means were found, means were separated using Duncan's multiple range test at $(P<0.05)$ (Duncan, 1955).

$$
Y_{i j k}=\mu+T_{i}+S_{j}+(T S)_{i j}+e_{i j k}
$$

Where; $\mathbf{Y}_{\mathrm{ij} \mathbf{K}}=$ Observations, $\boldsymbol{\mu}=$ General mean, $\mathbf{T}_{\mathbf{i}}=$ Temperature, $\mathbf{S}_{\mathbf{j}}=$ Strains, $(\mathbf{T S})_{\mathrm{ij}}=$ Interaction between three strains and different temperature and $\mathbf{e}_{\mathbf{i j k}}=$ Residual error.

\section{RESULTS AND DISCUSSION}

\section{Body weight and body weight gain}

Body weight of broiler chicks strains are presented in Table (1). Our study showed a highly significant difference between strains due to body weight. The Cobb hybrid recorded the highest weight at zero day at hatch $(46.07 \mathrm{~g})$ compared to Arbor Acres and Hubbard hybrids (41.39g \& 36.85g) subsequently.

The Cobb strain showed the best body weight during most of ages for each control and group which exposed to heat stress. Individuals of Cobb strain for control group recorded a significant difference values in body weight compared to another strains. It noticed that Cobb broiler strain reserve its performance under heat stress when compared to Arbor Acres and Hubbard broiler strains obviously which decreased in their body weight affected to heat stress exposure.

It seems that there is no significant effect for interaction between treatment effect and strain effect during all ages of flocks in this study. On the other hand, Altan et al (2000) decided metabolic and physiological responses of Hubbard and Cobb strains which exposed to an ambient temperature of
$38 \pm 1^{\circ} \mathrm{C}$ for 2 hour at 14 and 15 days of age. They found that exposure to high temperature at an early age resulted in weight loss in Cobb broiler strain which was not compensated at 35 days of age but there was no weight loss in Hubbard broiler strain.

Data presented in Table (2) showed that the live body weight gain for Cobb broiler strain recorded a heights value compared to Arbor Acres and Hubbard strains for cumulative body weight gain during the five weeks of age. It found that groups which exposed to heat stress recorded a lower values of body weight gain compared to the control group for the same strains under the same conditions of environment. It could be noticed that effect of (treatment*strain) interaction showed no significant differences during all week of age. Al-Batshan (2002) showed the effect of ambient temperature (33 \pm $0.5^{\circ} \mathrm{C}$ ) and genotype (Hubbard and IsAJ57) was evaluated in a factorial arrangement on broiler performance and found that high ambient temperature significantly decreased the body weight gain and the reduction under the hot temperature was more pronounced for Hubbard broiler chicks than those of the IsAJ57 strain.

\section{Carcass measurements}

Effect of both strain and heat exposure on inedible parts of carcass is summarized in Table (3). There was statistically significant difference obtained for feather weight $(122.6 \mathrm{~g})$ due to the effect of heat stress compared to strains which didn't exposed to heat stress which recorded (98.34 g). While, due to strain effect, the Hubbard strain recorded a non-significant decrease in feather weight (103.46 g) compared to Cobb (113.25 g) and Arbor Acres (114.69 g). Blood weight recorded non-statistically significant differences, which Hubbard strain recorded the highest non-significant value $(73.33 \mathrm{~g})$ compared to Cobb $(65.0 \mathrm{~g})$ and Arbor Acres strain $(66.47 \mathrm{~g})$.

In this study, Table (4) showed that gizzard weight recorded non-significant differences due to strain effect or heat exposure effect and interaction between them. The heart weight in Hubbard strain recorded the highest non-significant value $(8.485 \mathrm{~g})$ compared to Cobb $(9.18 \mathrm{~g})$ and Arbor Acres (9.285 g). Heat exposure effect caused a lower non-significant value $(8.35 \mathrm{~g})$ of heart weight compared to control groups $(9.1 \mathrm{~g})$.

Edible parts of chickens were weighted at five weeks of age for the three broiler strains under control conditions and heat exposed conditions. It could be observed that the strains which exposed to heat 
Table 1. Means and (SE) of live body weight for broiler strains as affected by heat stress exposure

\begin{tabular}{|c|c|c|c|c|c|c|c|}
\hline \multirow{2}{*}{ Age } & \multirow{2}{*}{ Strain } & \multicolumn{2}{|c|}{ Heat stress } & \multirow{2}{*}{ Overall (strain) } & \multicolumn{3}{|c|}{ Pr. } \\
\hline & & Control & Treated & & $\operatorname{Tr}$ & st & $\operatorname{tr}^{*} s t$ \\
\hline \multirow{4}{*}{$\begin{array}{c}0 \text { day } \\
\text { at hatch }\end{array}$} & & $\begin{array}{l}36.64 \\
\pm 0.43\end{array}$ & $\begin{array}{l}37.06 \\
\pm 0.34\end{array}$ & $36.85^{\mathrm{c}}$ & \multirow{4}{*}{$\begin{array}{l}\circ \\
\text { 옹 } \\
\text { 잉 }\end{array}$} & \multirow{4}{*}{$\begin{array}{l}\hat{\circ} \\
\text { ㅇㅇㅇ }\end{array}$} & \multirow{4}{*}{ 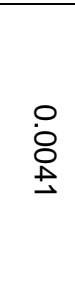 } \\
\hline & Cobb & $\begin{array}{l}44.74 \\
\pm 0.46\end{array}$ & $\begin{array}{l}47.40 \\
\pm 0.52\end{array}$ & $46.07^{a}$ & & & \\
\hline & Arbor Acres & $\begin{array}{l}41.38 \\
\pm 0.48 \\
\end{array}$ & $\begin{array}{l}41.22 \\
\pm 0.41 \\
\end{array}$ & $41.3^{b}$ & & & \\
\hline & Overall (trait) & $40.92^{\mathrm{b}}$ & $41.89^{a}$ & & & & \\
\hline \multirow{4}{*}{$1^{\text {st }} \mathrm{wk}$} & & $\begin{array}{l}153.42 \\
\pm 2.85\end{array}$ & $\begin{array}{c}137.48 \\
\pm 3.16\end{array}$ & $145.45^{b}$ & \multirow{4}{*}{$\begin{array}{l}\circ \\
\text { 응 } \\
\text { 인 }\end{array}$} & \multirow{4}{*}{$\begin{array}{l}\hat{\circ} \\
\text { 옹 }\end{array}$} & \multirow{4}{*}{ c) } \\
\hline & Cobb & $\begin{array}{c}169.12 \\
\pm 3.89\end{array}$ & $\begin{array}{c}158.96 \\
\pm 2.75\end{array}$ & $164.04^{a}$ & & & \\
\hline & Arbor Acres & $\begin{array}{c}151.46 \\
\pm 3.89 \\
\end{array}$ & $\begin{array}{c}147.30 \\
\pm 2.73 \\
\end{array}$ & $149.38^{b}$ & & & \\
\hline & Overall (trait) & $158^{a}$ & $147.91^{\mathrm{b}}$ & & & & \\
\hline \multirow{4}{*}{$2^{\text {nd }} w k$} & & $\begin{array}{c}389.84 \\
\pm 8.71\end{array}$ & $\begin{array}{c}376.51 \\
\pm 9.29\end{array}$ & & \multirow{4}{*}{ ¿ } & \multirow{4}{*}{$\begin{array}{l}\circ \\
\text { 영 }\end{array}$} & \multirow{4}{*}{ c } \\
\hline & Cobb & $\begin{array}{c}400.60 \\
\pm 8.51\end{array}$ & $\begin{array}{c}406.06 \\
\pm 8.62\end{array}$ & $403.33^{a}$ & & & \\
\hline & Arbor Acres & $\begin{array}{c}389.22 \\
\pm 7.52 \\
\end{array}$ & $\begin{array}{c}395.43 \\
\pm 7.55 \\
\end{array}$ & & & & \\
\hline & Overall (trait) & 393.22 & 392.67 & & & & \\
\hline \multirow{4}{*}{$3^{\text {rd }} \mathbf{w k}$} & & $\begin{array}{l}770.41 \\
\pm 16.92\end{array}$ & $\begin{array}{l}747.37 \\
\pm 17.36\end{array}$ & $758.89^{b}$ & \multirow{4}{*}{ C) } & \multirow{4}{*}{$\begin{array}{l}\circ \\
\text { 옹 }\end{array}$} & \multirow{4}{*}{ c) } \\
\hline & Cobb & $\begin{array}{l}819.36 \\
\pm 16.38\end{array}$ & $\begin{array}{l}839.04 \\
\pm 22.52\end{array}$ & $829.2^{a}$ & & & \\
\hline & Arbor Acres & $\begin{array}{r}766.18 \\
\pm 15.18 \\
\end{array}$ & $\begin{array}{l}722.14 \\
\pm 14.80 \\
\end{array}$ & $744.16^{b}$ & & & \\
\hline & Overall (trait) & 785.32 & 769.52 & & & & \\
\hline \multirow{4}{*}{$4^{\text {th }} w k$} & Hubbard & $\begin{array}{c}1185.78 \\
\pm 30.07\end{array}$ & $\begin{array}{c}1153.11 \\
\pm 44.46\end{array}$ & 1169.445 & \multirow{4}{*}{ c } & \multirow{4}{*}{ c } & \multirow{4}{*}{ ふ } \\
\hline & Cobb & $\begin{array}{l}1173.32 \\
\pm 29.12\end{array}$ & $\begin{array}{c}1213.70 \\
\pm 30.36\end{array}$ & 1193.51 & & & \\
\hline & Arbor Acres & $\begin{array}{c}1188.87 \\
\pm 31.61 \\
\end{array}$ & $\begin{array}{c}1100.81 \\
\pm 35.65 \\
\end{array}$ & 1144.84 & & & \\
\hline & Overall (trait) & 1182.66 & 1155.87 & & & & \\
\hline \multirow{4}{*}{$5^{\text {th }} \mathbf{w k}$} & Hubbard & $\begin{array}{c}1528.48 \\
\pm 38.27\end{array}$ & $\begin{array}{c}1487.41 \\
\pm 40.59\end{array}$ & $1507.945^{b}$ & \multirow{4}{*}{ c } & \multirow{4}{*}{$\begin{array}{l}\circ \\
\circ \\
\circ \\
\mathbb{1}\end{array}$} & \multirow{4}{*}{ ふ } \\
\hline & Cobb & $\begin{array}{c}1626.84 \\
\pm 39.64\end{array}$ & $\begin{array}{c}1640.81 \\
\pm 33.78\end{array}$ & $1633.825^{a}$ & & & \\
\hline & & $\begin{array}{c}1582.43 \\
\pm 43.52 \\
\end{array}$ & $\begin{array}{c}1495.75 \\
\pm 39.72 \\
\end{array}$ & $1539.09^{b}$ & & & \\
\hline & Overall (trait) & 1579.25 & 1541.32 & & & & \\
\hline
\end{tabular}

$a, b$ and $c$ Means within the same row with different letters are significantly differed. $\operatorname{Pr}=$ probability, NS = non-significant. $\operatorname{tr}=$ treatment, st $=$ strain. 
Table 2. Means and (SE) of live body weight gain for broiler strains as affected by heat stress exposure

\begin{tabular}{|c|c|c|c|c|c|c|c|}
\hline \multirow{2}{*}{ Age } & \multirow{2}{*}{ Strain } & \multicolumn{2}{|c|}{ Heat stress } & \multirow{2}{*}{ Overall (strain) } & \multicolumn{3}{|c|}{ Pr. } \\
\hline & & Control & Treated & & $\operatorname{tr}$ & st & $\operatorname{tr}^{*} s t$ \\
\hline \multirow{4}{*}{ 0-1 wk. } & Hubbard & $\begin{array}{l}116.78 \\
+279\end{array}$ & $\begin{array}{c}100.52 \\
+3.14\end{array}$ & $108.65^{b}$ & \multirow{4}{*}{$\hat{\dot{8}}$} & \multirow{4}{*}{$\begin{array}{l}0 \\
\dot{0} \\
\stackrel{\infty}{\infty} \\
0\end{array}$} & \multirow{4}{*}{ c) } \\
\hline & Cobb & $\begin{array}{c}124.36 \\
\pm 3.94\end{array}$ & $\begin{array}{c}111.57 \\
\pm 2.87\end{array}$ & $117.965^{a}$ & & & \\
\hline & Arbor Acres & $\begin{array}{c}110.08 \\
\pm 3.90 \\
\end{array}$ & $\begin{array}{c}106.08 \\
\pm 2.86 \\
\end{array}$ & $108.08^{b}$ & & & \\
\hline & Overall (trait) & $117.07^{a}$ & $106.05^{b}$ & & & & \\
\hline \multirow{4}{*}{ 1-2 wk. } & & $\begin{array}{c}236.42 \\
\pm 9.38\end{array}$ & $\begin{array}{c}237.46 \\
\pm 8.69\end{array}$ & 236.94 & \multirow{4}{*}{ ๘ } & \multirow{4}{*}{ ऊ } & \multirow{4}{*}{ z } \\
\hline & Cobb & $\begin{array}{c}232.53 \\
\pm 8.52\end{array}$ & $\begin{array}{c}247.10 \\
\pm 8.97\end{array}$ & 239.81 & & & \\
\hline & Arbor Acres & $\begin{array}{c}237.76 \\
\pm 8.47\end{array}$ & $\begin{array}{c}247.78 \\
\pm 7.64\end{array}$ & 242.77 & & & \\
\hline & Overall (trait) & 235.57 & 244.11 & & & & \\
\hline \multirow{4}{*}{ 2-3 wk. } & Hubbard & $\begin{array}{l}381.20 \\
\pm 17.68\end{array}$ & $\begin{array}{l}362.15 \\
\pm 18.81\end{array}$ & $371.67^{b}$ & \multirow{4}{*}{ ๘ } & \multirow{4}{*}{$\begin{array}{l}\text { ㅇ } \\
\text { ㅇ } \\
\dot{\omega} \\
+\end{array}$} & \multirow{4}{*}{ ¿ } \\
\hline & Cobb & $\begin{array}{l}428.47 \\
\pm 18.10\end{array}$ & $\begin{array}{r}432.00 \\
\pm 23.59\end{array}$ & $430.23^{a}$ & & & \\
\hline & Arbor Acres & $\begin{array}{l}376.37 \\
\pm 15.52\end{array}$ & $\begin{array}{l}381.13 \\
\pm 16.74\end{array}$ & & & & \\
\hline & Overall (trait) & 395.34 & 391.76 & & & & \\
\hline \multirow{4}{*}{ 3-4 wk. } & Hubbard & $\begin{array}{l}413.47 \\
\pm 36.23\end{array}$ & $\begin{array}{l}389.41 \\
\pm 41.19\end{array}$ & $401.44^{\mathrm{a}}$ & \multirow{4}{*}{ 웅 } & \multirow{4}{*}{$\begin{array}{l}0 \\
\dot{\circ} \\
\dot{p}\end{array}$} & \multirow{4}{*}{ ¿ } \\
\hline & Cobb & $\begin{array}{l}353.96 \\
\pm 33.74\end{array}$ & $\begin{array}{l}376.18 \\
\pm 36.92\end{array}$ & $365.07^{b}$ & & & \\
\hline & Arbor Acres & $\begin{array}{r}418.60 \\
\pm 35.44 \\
\end{array}$ & $\begin{array}{r}329.25 \\
\pm 35.48 \\
\end{array}$ & $373.92^{b}$ & & & \\
\hline & Overall (trait) & $395.34^{a}$ & $364.94^{b}$ & & & & \\
\hline \multirow{4}{*}{ 4-5 wk. } & Hubbard & $\begin{array}{l}337.97 \\
\pm 55.16\end{array}$ & $\begin{array}{l}334.71 \\
\pm 61.04\end{array}$ & $336.34^{c}$ & \multirow{4}{*}{ ๘ } & \multirow{4}{*}{ 오 } & \multirow{4}{*}{ ¿ } \\
\hline & Cobb & $\begin{array}{l}460.00 \\
\pm 45.68\end{array}$ & $\begin{array}{r}418.38 \\
\pm 39.74\end{array}$ & $439.19^{a}$ & & & \\
\hline & Arbor Acres & $\begin{array}{r}394.20 \\
\pm 47.87 \\
\end{array}$ & $\begin{array}{r}388.55 \\
\pm 54.99 \\
\end{array}$ & $391.37^{b}$ & & & \\
\hline & Overall (trait) & 397.39 & 380.54 & & & & \\
\hline \multirow{4}{*}{$\begin{array}{c}\text { Cumulative } \\
\text { BWG } \\
\text { 0-5 wk. }\end{array}$} & Hubbard & $\begin{array}{c}1492.18 \\
\pm 38.13\end{array}$ & $\begin{array}{c}1450.62 \\
\pm 40.56\end{array}$ & $1471.40^{\mathrm{b}}$ & \multirow{4}{*}{ ๘ } & \multirow{4}{*}{$\begin{array}{l}\circ \\
\circ \\
\varnothing \\
\varnothing\end{array}$} & \multirow{4}{*}{ क } \\
\hline & Cobb & $\begin{array}{c}1582.11 \\
\pm 39.44\end{array}$ & $\begin{array}{c}1593.49 \\
\pm 33.87\end{array}$ & $1587.80^{a}$ & & & \\
\hline & Arbor Acres & $\begin{array}{c}1541.09 \\
\pm 43.56 \\
\end{array}$ & $\begin{array}{c}1454.85 \\
\pm 39.75 \\
\end{array}$ & $1497.97^{b}$ & & & \\
\hline & Overall (trait) & 1538.46 & 1499.65 & & & & \\
\hline
\end{tabular}

$a, b$ and $c$ Means within the same row with different letters are significantly differed. $\mathrm{Pr}=$ probability, NS = non-significant. $\mathrm{tr}=$ treatment, $\mathrm{st}=$ strain. 
Table 3. Inedible parts of carcass traits for broiler strains as affected by heat stress exposure

\begin{tabular}{|c|c|c|c|c|c|c|c|}
\hline \multirow{2}{*}{ Item } & \multirow{2}{*}{ Strain } & \multicolumn{2}{|c|}{ Heat stress } & \multirow{2}{*}{$\begin{array}{l}\text { Overall } \\
\text { (strain) }\end{array}$} & \multicolumn{3}{|c|}{ Pr. } \\
\hline & & Control & Treated & & $\operatorname{tr}$ & st & $\operatorname{tr}^{\star} s t$ \\
\hline \multirow{5}{*}{ Live Body weight (g.) } & Hubbard & 1995.00 & 2022.00 & 2008.5 & \multirow{5}{*}{ ¿ } & \multirow{5}{*}{ ふ } & \multirow{5}{*}{ z } \\
\hline & & -61.00 & -100.02 & & & & \\
\hline & & $\begin{array}{c}2068.75 \\
\pm 81.23\end{array}$ & $\begin{array}{c}1848.75 \\
\pm 53.41\end{array}$ & 1958.75 & & & \\
\hline & Arbor Acres & $\begin{array}{c}1951.25 \\
\pm 57.86 \\
\end{array}$ & $\begin{array}{c}2015.63 \\
\pm 72.87 \\
\end{array}$ & 1983.305 & & & \\
\hline & Overall (trait) & 2005.00 & 1962.13 & & & & \\
\hline \multirow{6}{*}{$\begin{array}{c}\text { Blood } \\
\text { Weight (g.) }\end{array}$} & Hubbard & $\begin{array}{c}79.38 \\
\pm 15.82\end{array}$ & $\begin{array}{c}67.29 \\
\pm 11.99\end{array}$ & 73.335 & \multirow{6}{*}{ ऊ } & \multirow{6}{*}{ そ } & \multirow{6}{*}{ ¿ } \\
\hline & Cobb & 64.38 & 65.63 & 65.005 & & & \\
\hline & & \pm 4.17 & \pm 5.38 & & & & \\
\hline & Arbor Acres & 64.38 & 68.57 & 66.475 & & & \\
\hline & & \pm 5.04 & \pm 7.54 & & & & \\
\hline & Overall (trait) & 69.38 & 67.16 & & & & \\
\hline \multirow{4}{*}{ Feathers weight (g.) } & & $\begin{array}{c}86.66 \\
\pm 13.33\end{array}$ & $\begin{array}{c}120.26 \\
\pm 7.83\end{array}$ & 103.46 & \multirow{4}{*}{$\begin{array}{l}0 \\
\stackrel{0}{8} \\
\stackrel{1}{+}\end{array}$} & \multirow{4}{*}{ そ } & \multirow{4}{*}{ z } \\
\hline & Cobb & $\begin{array}{c}107.10 \\
\pm 6.65\end{array}$ & $\begin{array}{l}119.40 \\
\pm 11.17\end{array}$ & 113.25 & & & \\
\hline & Arbor Acres & $\begin{array}{c}101.25 \\
\pm 8.28 \\
\end{array}$ & $\begin{array}{r}128.13 \\
\pm 11.06 \\
\end{array}$ & 114.69 & & & \\
\hline & Overall (trait) & $98.34^{b}$ & $122.60^{a}$ & & & & \\
\hline \multirow{6}{*}{$\begin{array}{c}\text { Head } \\
\text { Weight (g.) }\end{array}$} & Hubbard & 43.20 & 40.93 & 42065 & \multirow{6}{*}{$\begin{array}{l}\circ \\
\stackrel{\circ}{+} \\
\stackrel{+}{\rightarrow}\end{array}$} & \multirow{6}{*}{ ふ } & \multirow{6}{*}{ ¿ } \\
\hline & & \pm 1.67 & \pm 1.74 & & & & \\
\hline & Cobb & 44.62 & 37.71 & 41.165 & & & \\
\hline & & \pm 2.00 & \pm 1.50 & & & & \\
\hline & Arbor Acres & $\begin{array}{r}44.35 \\
\pm 0.96\end{array}$ & $\begin{array}{l}43.95 \\
\pm 2.41\end{array}$ & 44.15 & & & \\
\hline & Overall (trait) & $44.06^{\mathrm{a}}$ & $40.86^{b}$ & & & & \\
\hline \multirow{7}{*}{ Two legs weight (g.) } & Hubhord & 69.31 & 72.37 & 7094 & \multirow{7}{*}{ क } & \multirow{7}{*}{ c } & \multirow{7}{*}{ c } \\
\hline & Thunara & \pm 2.59 & \pm 4.79 & 10.04 & & & \\
\hline & Cobh & 81.80 & 71.03 & 76.415 & & & \\
\hline & 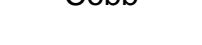 & \pm 4.26 & \pm 2.76 & & & & \\
\hline & $\hat{x}$ rhore 1 & 72.92 & 70.18 & 7106 & & & \\
\hline & Aroor & \pm 3.45 & \pm 3.67 & 11.865 & & & \\
\hline & Overall (trait) & 83.68 & 71.19 & & & & \\
\hline
\end{tabular}

$a, b$ and $c$ Means within the same row with different letters are significantly differed. $\mathrm{Pr}=$ probability, $\mathrm{NS}=$ non-significant. $\operatorname{tr}=$ treatment, $s t=$ strain. 
Table 4. Edible parts of carcass traits for broiler strains as affected by heat stress exposure

\begin{tabular}{|c|c|c|c|c|c|c|c|}
\hline \multirow{2}{*}{ Age } & \multirow{2}{*}{ Strain } & \multicolumn{2}{|c|}{ Heat stress } & \multirow{2}{*}{$\begin{array}{l}\text { Overall } \\
\text { (strain) }\end{array}$} & \multicolumn{3}{|c|}{ Pr. } \\
\hline & & Control & Treated & & $\operatorname{tr}$ & st & $\operatorname{tr}^{*} s t$ \\
\hline \multirow{4}{*}{$\begin{array}{c}\text { Dressing } \\
\text { Weight (g.) }\end{array}$} & Hubbard & $1455.61 \pm 33.18$ & $1436.63 \pm 79.99$ & 1446.12 & \multirow{4}{*}{ 品 } & \multirow{4}{*}{ co } & \multirow{4}{*}{ co } \\
\hline & Cobb & $1503.52 \pm 69.08$ & $1329.13 \pm 50.19$ & 1416.32 & & & \\
\hline & Arbor Acres & $1454.31 \pm 42.92$ & $1442.15 \pm 74.77$ & 1448.23 & & & \\
\hline & Overall (trait) & $1471.15^{a}$ & $1402.64^{b}$ & & & & \\
\hline \multirow{4}{*}{$\begin{array}{l}\text { Major Breast } \\
\text { Muscle } \\
\text { Weight (g.) }\end{array}$} & Hubbard & $153.05 \pm 5.73$ & $163.80 \pm 10.52$ & $158.42^{a}$ & \multirow{4}{*}{ zo } & \multirow{4}{*}{ 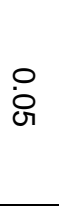 } & \multirow{4}{*}{ m } \\
\hline & Cobb & $157.61 \pm 9.81$ & $131.15 \pm 5.47$ & $144.38^{b}$ & & & \\
\hline & Arbor Acres & $153.93 \pm 6.74$ & $166.00 \pm 10.98$ & $159.96^{\mathrm{a}}$ & & & \\
\hline & Overall (trait) & 154.86 & 153.65 & & & & \\
\hline \multirow{4}{*}{$\begin{array}{c}\text { Minor Breast } \\
\text { Muscle } \\
\text { Weight (g.) }\end{array}$} & Hubbard & $39.31 \pm 3.69$ & $39.29 \pm 3.01$ & $39.30^{\mathrm{b}}$ & \multirow{4}{*}{ z } & \multirow{4}{*}{$\begin{array}{l}\text { Oे } \\
\dot{O}\end{array}$} & \multirow{4}{*}{ c } \\
\hline & Cobb & $39.59 \pm 1.62$ & $32.30 \pm 1.04$ & $35.94^{a}$ & & & \\
\hline & Arbor Acres & $36.93 \pm 2.31$ & $41.66 \pm 2.34$ & $39.29^{b}$ & & & \\
\hline & Overall (trait) & 38.61 & 37.75 & & & & \\
\hline \multirow{4}{*}{$\begin{array}{c}\text { Thigh Muscle } \\
\text { Weight (g.) }\end{array}$} & Hubbard & $163.73 \pm 6.47$ & $164.65 \pm 10.83$ & $164.19^{b}$ & \multirow{4}{*}{ zo } & \multirow{4}{*}{$\begin{array}{l}\text { 웅 } \\
\text { c }\end{array}$} & \multirow{4}{*}{ c } \\
\hline & Cobb & $184.23 \pm 11.88$ & $176.22 \pm 7.96$ & $180.22 a$ & & & \\
\hline & Arbor Acres & $153.66 \pm 8.79$ & $175.90 \pm 10.51$ & $164.78 \mathrm{~b}$ & & & \\
\hline & Overall (trait) & 167.21 & 172.26 & & & & \\
\hline \multirow{4}{*}{$\begin{array}{l}\text { Drum stick } \\
\text { Weight (g.) }\end{array}$} & Hubbard & $93.43 \pm 3.39$ & $101.60 \pm 6.32$ & 97.51 & \multirow{4}{*}{ zo } & \multirow{4}{*}{ c } & \multirow{4}{*}{ z } \\
\hline & Cobb & $101.45 \pm 5.69$ & $82.32 \pm 5.06$ & 91.88 & & & \\
\hline & Arbor Acres & $92.21 \pm 2.01$ & $94.39 \pm 6.86$ & 93.30 & & & \\
\hline & Overall (trait) & 95.70 & 92.77 & & & & \\
\hline \multirow{4}{*}{$\begin{array}{l}\text { Liver weight } \\
\text { (g.) }\end{array}$} & Hubbard & $52.07 \pm 1.74$ & $53.08 \pm 2.67$ & 52.57 & \multirow{4}{*}{ z } & \multirow{4}{*}{ z } & \\
\hline & Cobb & $51.57 \pm 2.04$ & $48.18 \pm 2.65$ & 49.87 & & & $z$ \\
\hline & Arbor Acres & $51.91 \pm 2.06$ & $47.44 \pm 3.68$ & 49.67 & & & \\
\hline & Overall (trait) & 51.85 & 49.57 & & & & \\
\hline & Hubbard & $27.47 \pm 2.01$ & $23.87 \pm 1.53$ & 25.67 & & & \\
\hline Gizzard weight & Cobb & $24.15 \pm 1.08$ & $24.84 \pm 2.51$ & 24.49 & $z$ & $z$ & $z$ \\
\hline (g.) & Arbor Acres & $22.86 \pm 1.30$ & $25.01 \pm 1.66$ & 23.93 & & & \\
\hline & Overall (trait) & 24.83 & 24.57 & & & & \\
\hline & Hubbard & $8.13 \pm 0.49$ & $8.84 \pm 0.47$ & 8.48 & & & \\
\hline Heart weight & Cobb & $9.70 \pm 0.56$ & $8.66 \pm 0.58$ & 9.18 & $z$ & $z$ & $z$ \\
\hline (g.) & Arbor Acres & $9.47 \pm 0.57$ & $9.10 \pm 0.06$ & 9.28 & & & \\
\hline & Overall (trait) & 9.10 & 8.35 & & & & \\
\hline
\end{tabular}

$a, b$ and $c$ Means within the same row with different letters are significantly differed. $\mathrm{Pr}=$ probability, NS = non-significant. $\operatorname{tr}=$ treatment, st= strain. 
stress recorded slightly non-significant value for dressing weight compared to control strains. We indicated that Cobb broiler strain recorded the lowest value for dressing weight under effect of heat stress compared to another strains. Conversely, the Cobb strain recorded the highest value for dressing weight under the control conditions of environmental temperature.

The different muscles (major breast muscles, minor breast muscles, thigh and drums stick) weights showed slightly non-significant a differences with effect of strains and also the same results with effect of heat exposure. But Cobb strain showed lower major and minor breast muscle weight (Pr st $=0.05$ ) compared to Hubbard and Arbor Acres strains as well as higher (Pr st $=0.05)$ thigh muscle weight than Hubbard and Arbor Acres strains.

Data summarized in Table (5) indicated that there were non-significant differences for bursa weight affected with treatment, strain and interaction between them. The control group showed an increase in bursa weight $(3.68 \mathrm{~g})$, while the exposed groups recorded (3.29 g). Hubbard strain recorded the higher non-significant value (NS = Pr st) of bursa weight (3.69 g) compared to Cobb $(3.4 \mathrm{~g})$ and Arbor Acres $(3.37 \mathrm{~g})$. Jovanirlnês Müller Fernandes et al (2013) showed that carcass yield had no significant effect registered in relation totemperature ofenvironment or heat stress during the last week of bird life. These results are probably associated with the small variation between temperatures used in the first week of bird life, not allowing acclimatization of these birds and not influencing thus physiological responses to heat stress applied on the last week of life. Gholam-Reza Zaboli et al (2016) found that the relative weight of breast (without skin), legs, liver and gizzard didn't differ among the experimental groups. The thermal manipulation caused a decrease in the relative weight of the heart. Table 5 also shows a higher spleen weight for Arbor Acres strain (Pr st $=0.05$ ) compared to Cobb strain.

\section{Rectal Temperature}

Data summarized in Figs. (1\&2) showed the rectal temperature for the three strains which exposed to heat stress and another control groups. At one day of age Arbor Acres strain showed a narrow range between $\mathrm{RT}$ for control group $\left(40.85^{\circ} \mathrm{C}\right)$ and heat affected groups $\left(41.19^{\circ} \mathrm{C}\right)$. While both (Hubbard \& Cobb) strains showed a wide range between RT for control group and heat exposed groups. Subsequently, the Arbor Acres considered the best strain which didn't effected a lot such another strains with heat exposure.

Table 5. Lymphoid organs weight for broiler strains as affected by heat stress exposure

\begin{tabular}{|c|c|c|c|c|c|c|c|}
\hline \multirow{2}{*}{ Item } & \multirow{2}{*}{ Strain } & \multicolumn{2}{|c|}{ Heat stress } & \multirow{2}{*}{$\begin{array}{l}\text { Overall } \\
\text { (strain) }\end{array}$} & \multicolumn{3}{|c|}{ Pr. } \\
\hline & & Control & Treated & & $\operatorname{tr}$ & st & $\operatorname{tr}^{*} s t$ \\
\hline \multirow{4}{*}{$\begin{array}{c}\text { Spleen weight } \\
\text { (g.) }\end{array}$} & Hubbard & $2.49 \pm 0.28$ & $2.11 \pm 0.23$ & $2.3^{a b}$ & \multirow{4}{*}{ ¿ } & \multirow{4}{*}{$\begin{array}{l}\circ \\
\text { 이 }\end{array}$} & \multirow{4}{*}{ ¿ } \\
\hline & Cobb & $1.68 \pm 0.19$ & $1.69 \pm 0.22$ & $1.69^{b}$ & & & \\
\hline & Arbor Acres & $2.16 \pm 0.20$ & $2.65 \pm 0.62$ & $2.41^{\mathrm{a}}$ & & & \\
\hline & Overall (trait) & 2.11 & 2.15 & & & & \\
\hline \multirow{4}{*}{$\begin{array}{c}\text { Bursa weight } \\
\text { (g.) }\end{array}$} & Hubbard & $3.27 \pm 0.73$ & $4.10 \pm 0.51$ & 3.69 & \multirow{4}{*}{ ¿ } & \multirow{4}{*}{ ふ } & \multirow{4}{*}{ Z } \\
\hline & Cobb & $4.25 \pm 0.53$ & $2.54 \pm 0.36$ & 3.40 & & & \\
\hline & Arbor Acres & $3.51 \pm 0.30$ & $3.22 \pm 0.82$ & 3.37 & & & \\
\hline & Overall (trait) & 3.68 & 3.29 & & & & \\
\hline \multirow{4}{*}{$\begin{array}{c}\text { Thymus weight } \\
\text { (g.) }\end{array}$} & Hubbard & $3.78 \pm 0.99$ & $1.79 \pm 0.21$ & 2.79 & \multirow{4}{*}{ ฉ } & \multirow{4}{*}{ ऽ } & \multirow{4}{*}{ z } \\
\hline & Cobb & $1.78 \pm 0.25$ & $1.64 \pm 0.60$ & 1.71 & & & \\
\hline & Arbor Acres & $1.94 \pm 0.35$ & $1.86 \pm 0.54$ & 1.90 & & & \\
\hline & Overall (trait) & 2.50 & 1.76 & & & & \\
\hline
\end{tabular}

$a, b$ and $c$ Means within the same row with different letters are significantly differed. $\mathrm{Pr}=$ probability, NS = non-significant. $\operatorname{tr}=$ treatment, $\mathrm{st}=$ strain. 


\section{Rectal temp. at 1 day of age $\left({ }^{\circ} \mathrm{C}\right)$}

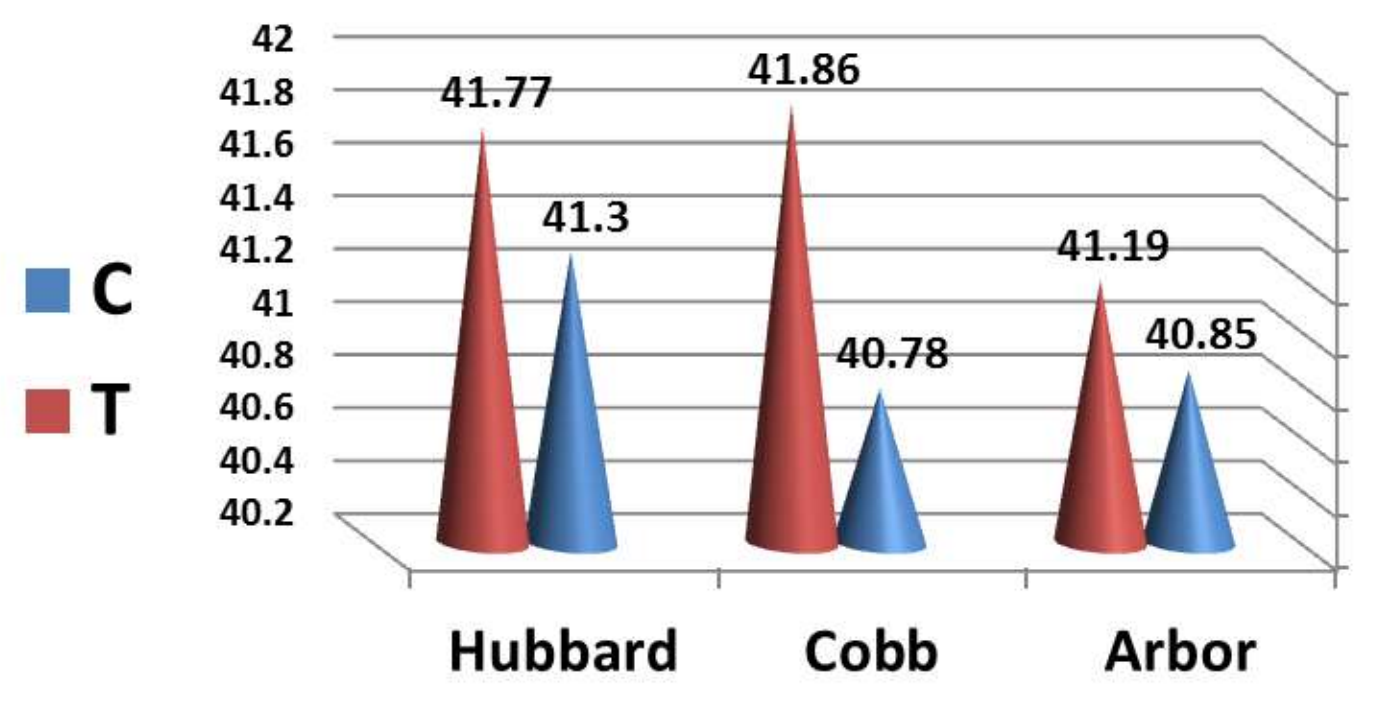

Fig. 1. The rectal temperature for the three strains which exposed to heat stress and control groups at first day after hatch $(P=0.0001$, for strain effect) and $(P<.0001$, for treatment effect)

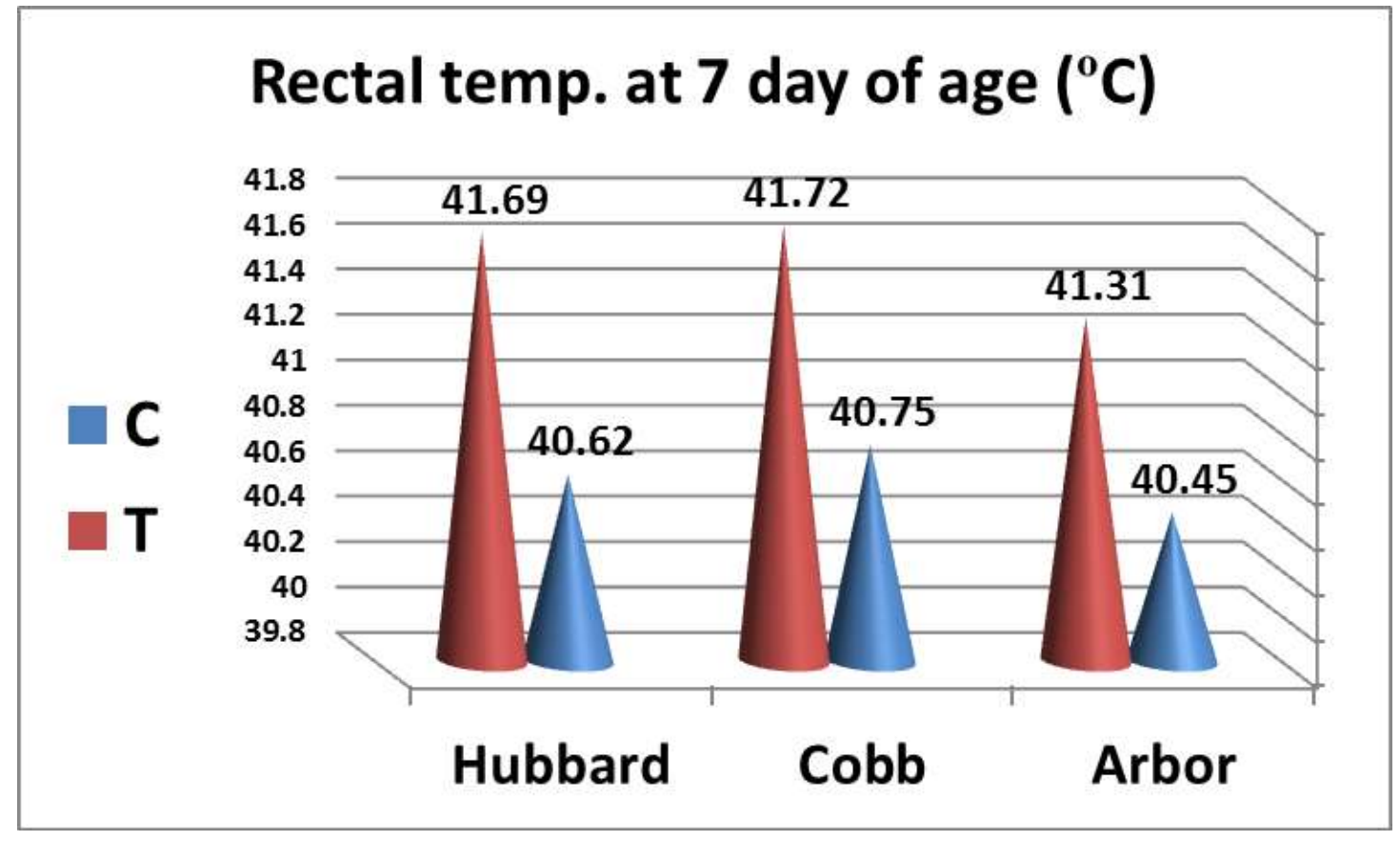

Fig. 2. The rectal temperature for the three strains which exposed to heat stress and control groups at the $7^{\text {th }}$ day after hatch. $(P=0.0407$, for strain effect $) \&(P<.0001$, for treatment effect $)$ 


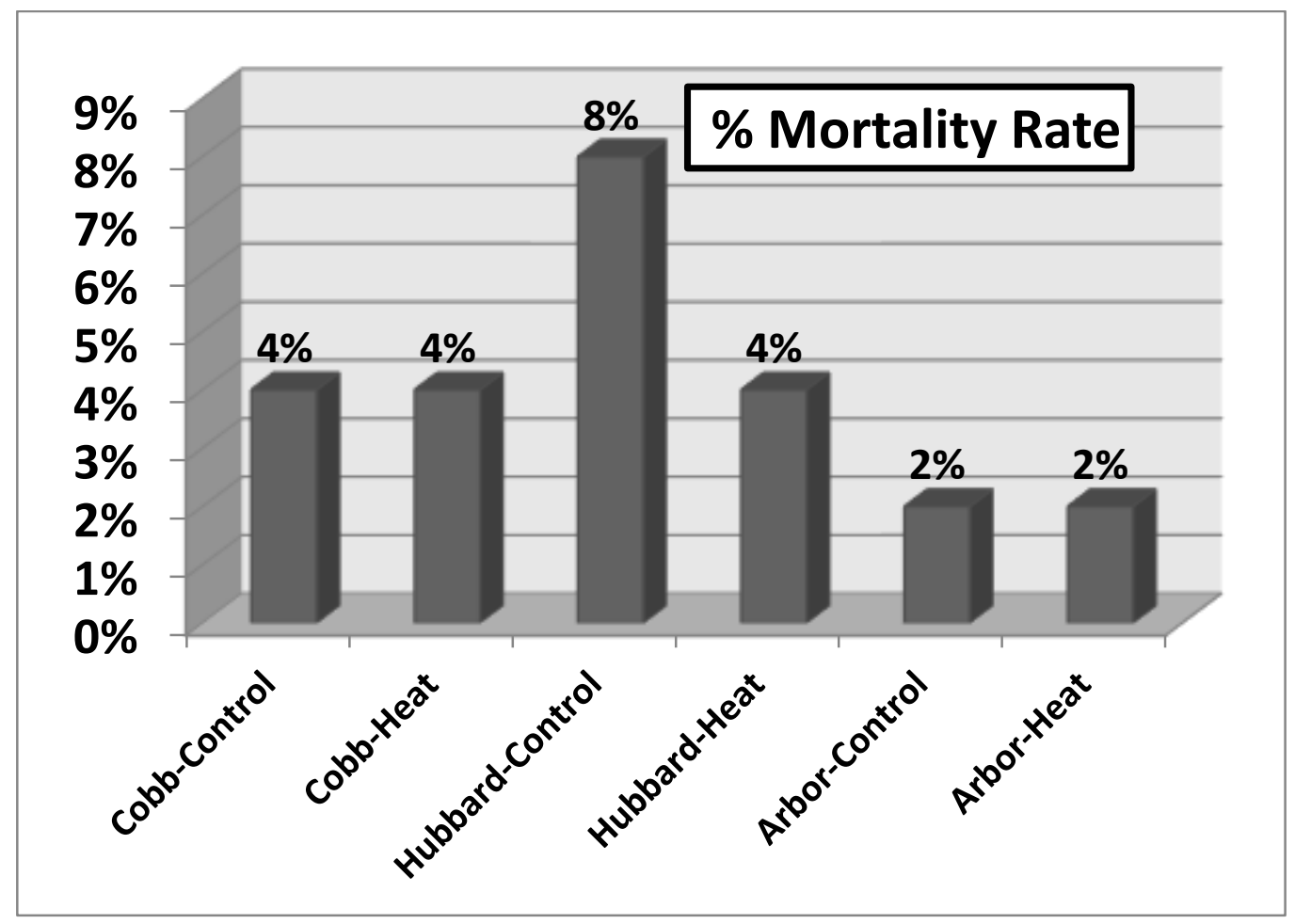

Fig. 3. The mortality rates for the three broiler hybrids under control and heat stress exposure

The same results were revealed at one week of age and Arbor Acres strain proved that it is the best for tolerance heat stress when comparing the range of rectal temperature for control and treated groups. Abioja et al (2014) showed that temperature of rectum used as a major indicator of heat stress in poultry (Abioja et al 2012). Heat stress leads to elevated body temperature as the environmental temperature shoots ahead of the comfort zone of the birds (Kumar et al 2011). Hiroshi Tanizawa et al (2014) reported that before the acute heat challenge, rectal temperature in control and treated chicks was $41.6^{\circ} \mathrm{C}$ and $41.7^{\circ} \mathrm{C}$, respectively. The temperatures of both groups were elevated by acute heat stress but that of the experienced chicks was significantly lower than control chicks (control: $43.09^{\circ} \mathrm{C} \&$ treatment: $42.87^{\circ} \mathrm{C}$ ).

\section{Mortality rate}

In our study Fig. (3) proved that the Arbor Acres strain for each group (control and treated) have the highest viability with mortality percentage ( $2 \%)$ for both of them while the control group of Hubbard strain showed the lowest viability with mortality percentage $(8 \%)$ may be to the highly body weight gain than another strains during period of (3 to 4 wk of age) with synchronize of exposure of environmental temperature.

\section{REFERENCES}

Abioja M.O., Sodipe O.G., Abiona J.A., Oladipo K.A., Kasali O.D., Akerele Z., Ola A., Oke H., Ogundele O. and Osinowo O.A. 2014. Thermotolerance Acquisition in Broiler Chickens through Early Feed Restriction: Response to Acute Heat Stress. Pertanika. J. Trop. Agric. Sci. 37(3), 311-319.

Abioja M.O., Ogundimu K.B., Akibo T.E., Odukoya K.E., Ajiboye O.O., Abiona J.A., Williams T.J., Oke E.O. and Osinowo O.A. 2012. Growth, mineral deposition and physiological responses of broiler chickens offered honey in drinking water during hot-dry season. Int. J. of Zoology, ISSN: 1687-8477; Volume: 2012. 
Al-Batshan 2002. Performance and heat tolerance of broilers as affected by genotype and high ambient temperature. Asian-Aust. J. Anim. Sci., 15(10), 1502-1506.

Altan O., Ali A., Metin Ç. and Hakan B. 2000. Effects of Heat Stress on Some Blood Parameters in Broilers. Turkish J. of Veterinary and Animal Sci., 24(2), 145-148.

Arce-Menocal J., Avila-Gonzalez E., LopezCoello C., Garibay Torres L. and MartinezLemus L.A. 2009. Body weight, feed particle size, and ascites incidence revisited. J. of Applied Poultry Research, 18, 465-471.

DOI:https://doi.org/10.3382/japr.2008-00095.

Dozier W.A., Purswell J., Kidd M.T., Corzo A. and Branton S.L. 2007. Apparent metabolizable energy needs of broilers from 2.0 to $4.0 \mathrm{~kg}$ as influenced by ambient temperature. J. of Applied. Poultry Research, 16, 206-218. DOI: 10.3382/ps.2010-01132

Duncan D.B. 1955. Multiple Range and Multiple F test. Biometrics, 11, 1-42.

Gholam-Reza Z., Shaban R., Farid S., Mohammad A.K.T., Ali B. and Mehran M. 2016. Thermal manipulation during Pre and Post-Hatch on thermotolerance of male broiler chickens exposed to chronic heat stress. Poultry Sci., 00, 1-88.

Havenstein G.B., Ferket P.R. and Qureshi M.A. 2003. Carcass composition and yield of 1957 versus 2001 broilers when fed representative 1957 and 2001 broiler diets. Poultry Sci., 82, 1509-1518. DOI: 10.1093/ps/82.10.1509.

Hiroshi T., Jun-ichiShiraishi, Shin-Ichi K., Masaoki T. and Takashi B. 2014. Effect of Short-Term Thermal Conditioning on Physiological and Behavioral Responses to Subsequent Acute Heat Exposure in Chicks. J. Poultry Sci., $51,80-86$.
Jovanir I.M.F., Lidiane B.S., Elisangela T.G., Alvaro M.B.J., Felipe E.D.S.M. and Leonardo G. 2013. Thermal conditioning during the first week on performance, heart morphology and carcass yield of broilers submitted to heat stress.

ActaScientiarum. Animal Sci. Maringá, 35(3), 311-319.

Kumar B.V.S., Kumar A. and Kataria M. 2011. Effect of heat stress in tropical livestock and different strategies for its amelioration. J. Stress PhysiolBiochem 7(1), 45-54.

Lourens A., van den Brand H., Meijerhof R. and Kemp B. 2005. Effect of eggshell temperature during incubation on embryo development, hatchability and post-hatch development. Poultry Sci. 84, 914-920.

Pourreza J. and Edriss M.A. 1992. The effects of high vs. normal temperatures on the physical characteristics of the broilers carcass. J. Agric. Sci. Technol. 1, 35-41.

Reddy C. 2000. Features Maintaining Growth and Production-Overcoming heat stress. Poultry Int., 39(2), 36-41.

Swayne H.J. Barnes J.R. Glisson and Fadly A.M. 2017. Disease of Poultry.13th. Press, ABlackwell Publishing Co Ames, lowa Thigpen. pp. 1056-1057.

Yousaf A., Tabasam M.S., Memon A., Rajput N., Shahnawaz R., Rajpar S., Jamil T. and Mushtaq M. 2019. Prevalence of ascaridiagalli in different broiler poultry farms of potohar region of rawalpindi-pakistan. J. of Dairy Veterinary Animals Research, 8(1), 71-73. DOI: 10.15406/jdvar.2019.08.00245

Yousaf A., Shahnawaz R., Jamil T. and Mushtaq A. 2018. Prevalence of coccidiosis in different broiler poultry farms in Potohar region (distract Rawalpindi) of Punjab- Pakistan. J. of Dairy Veterinary Animals Research, 7(3), 87-90. DOI:10.15406/jdvar.2018.07.00196. 


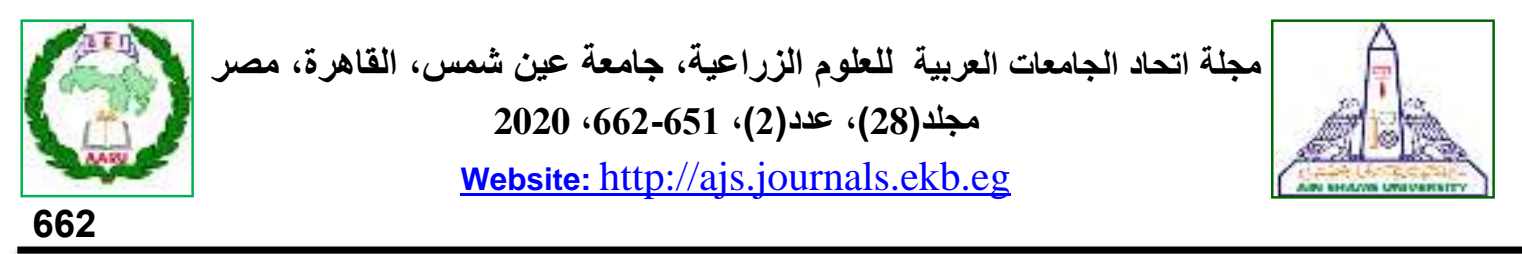

تأثير الإجهاد الحراري علي أداء النمو وصفات الذبيحة في بعض سلالات دجاج اللحم

[48]

$$
\begin{aligned}
& \text { عمر محمد عمران" - أحمد جلال - محمود يوسف محروس - فيصل بيومي بدري }
\end{aligned}
$$

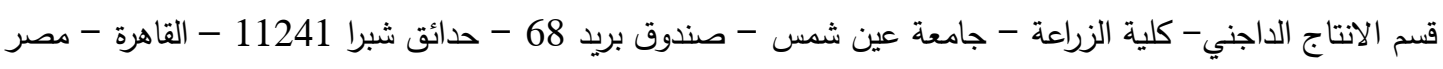

*Corresponding author: omeromran1987@gmail.com

Received 16 May, 2020

Accepted 19 August, 2020

درجة سليزيوس 2 د 2 درجة سليزيوس، رطوبة نسبية =

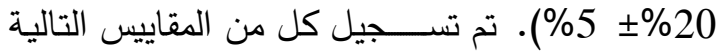

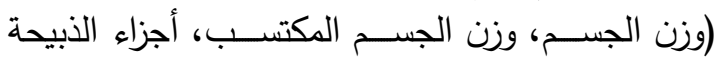

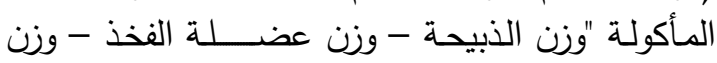

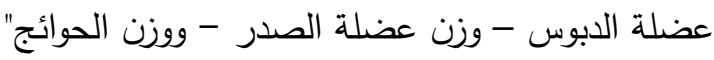

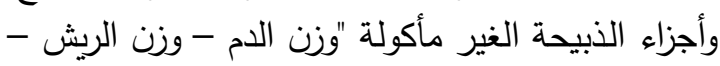

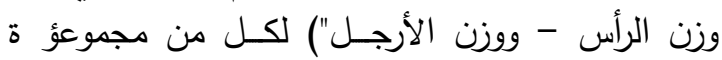

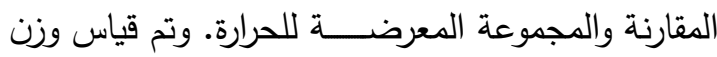

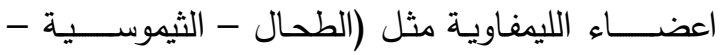

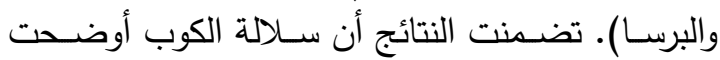

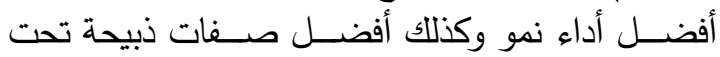
ظروف الإجهاد الحراري، بينما أعتبرت ســـلالة الآربور

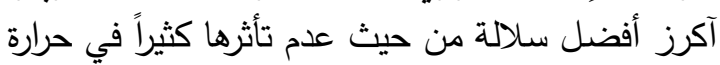

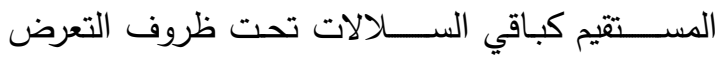

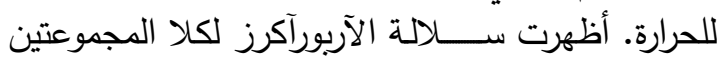

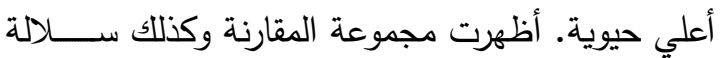

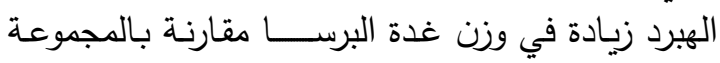

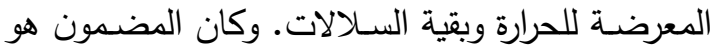

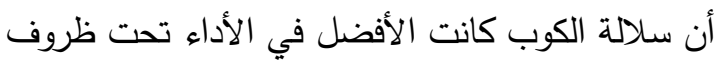
الإجهاد الحراري مقارنة بسلالات دجاج اللحم الأخري.

الكلمـات المفتاحية: دجاج التســـمين، النمو، الذبيحة، التعرض للحرارة

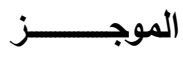

يعتبر الإجهاد الحراري للبيئة أحـد أكثر الظروف

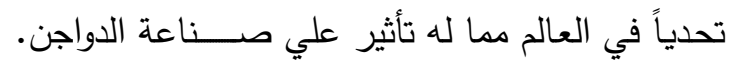

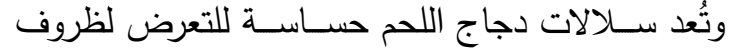

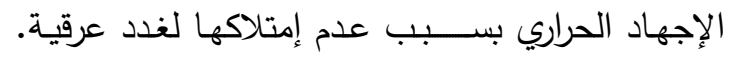

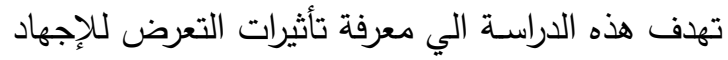

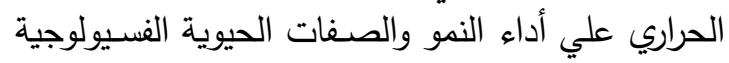

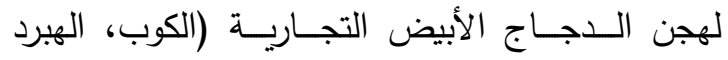

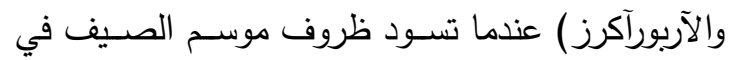

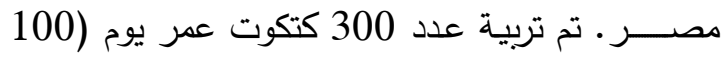
طائر من كل هجين) تحت نفس الظروف من استهالاك

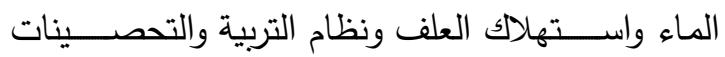

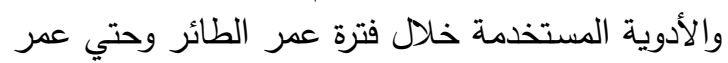

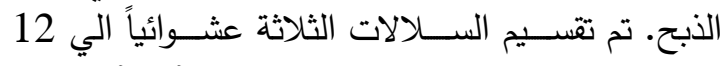

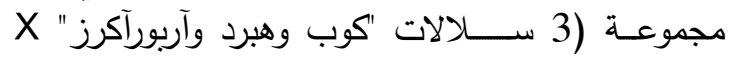

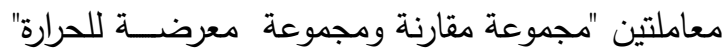

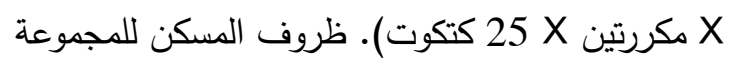

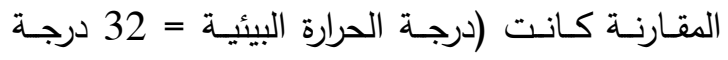

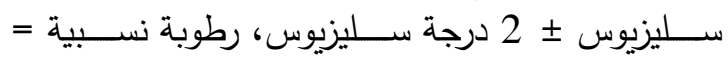

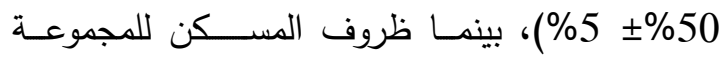
المعرضــــة للحرارة كانت (درجة الحرارة البيئية = 40

تحكيم: ا.د أميره الدلب

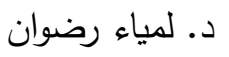

أجنبى 